\title{
Xanthine oxidase inhibitory and antioxidant potential of Indian Muscodor species
}

\author{
Neha Kapoor ${ }^{1} \cdot$ Sanjai Saxena $^{1}$ (D)
}

Received: 27 September 2016/Accepted: 11 November 2016/Published online: 17 November 2016

(c) The Author(s) 2016. This article is published with open access at Springerlink.com

\begin{abstract}
Xanthine oxidase is a key enzyme responsible for hyperuricemia, a pre-disposing factor for Gout and oxidative stress-related diseases. Only two clinically approved xanthine oxidase inhibitors Allopurinol and Febuxostat are currently used for treatment of hyperuricemia. However, owing to their side effects there is a need for new non-purine-based selective inhibitors of xanthine oxidase. In the process of exploring novel xanthine oxidase inhibitors and anti-oxidants, we screened the culture filtrate of 07 novel species of Muscodor, a sterile endophytic fungi isolated from Cinnamomum and Aegle marmelos. Chloroform extract of $M$. darjeelingensis exhibited the maximum xanthine oxidase inhibition in the qualitative and quantitative assays. The $\mathrm{IC}_{50}$ of chloroform extract of $M$. darjeelingensis was $0.54 \mu \mathrm{g} / \mathrm{ml}$ which was much lower to Allopurinol but higher when compared to Febuxostat. $88 \%$ reduction in uric acid production was recorded by $M$. darjeelingensis chloroform extract which was similar to allopurinol. The maximum anti-oxidant activity was exhibited by $M$. indica against the gallic acid standard in the DPPH-free radical assay. Anti-oxidant activity index of $M$. indica was 7.7, which was followed by M. kashayum with 5.4. M. darjeelingensis exhibited a moderate anti-oxidant activity with anti-oxidant activity index of 1.63 in the DPPH assay. The present study is the very first report of Muscodor species exhibiting xanthine oxidase inhibitory and anti-oxidant activity together. Chloroform extract of $M$. darjeelingensis and $M$. indica stand out as potential candidates for isolation and
\end{abstract}

Sanjai Saxena

sanjaibiotech@yahoo.com; ssaxena@thapar.edu

1 Department of Biotechnology, Thapar University, Patiala, Punjab 147004, India characterization of the xanthine oxidase inhibitor and antioxidant compound, respectively.

Keywords Endophytic fungi - DPPH assay · Enzyme inhibitor · Muscodor · Hyperuricemia

\section{Introduction}

Hyperuricemia is a pre-disposing factor of gout which has been recognized as a lifestyle disease affecting adult population in the developed as well as developing countries (Kuo et al. 2015). Hyperuricemia results due to high serum urate levels which is attributed to it's over production or under-excretion. The therapeutic strategies of treatment of hyperuricemia are by excretion of excessive uric acid or blocking the uric acid production. The later strategy appears to be safer since it involves the inhibition of Xanthine oxidase (XO), the key enzyme responsible for the production of uric acid. Till date, only Allopurinol and Febuxostat have been clinically approved as XO inhibitors for the treatment of hyperuricemia and gout. However, there is a demand for new non-purine-based selective inhibitors of Xanthine oxidase (NP-SIXO's) owing to the side effects of currently used drugs.

Over last two decades, endophytic fungi have been well recognized as fountainheads of novel bioactive compounds possessing anti-cancer, anti-microbial, and anti-oxidant properties as well as putative sources of phytochemicals. However, exploration of these microorganisms for NPSIXO's is very limited as evident from the literature (Kapoor and Saxena 2014).

The genus Muscodor emerged with the discovery of sterile endophytic fungus Muscodor albus from the branch of cinnamon plant in Honduras (Worapong et al. 2001). 
Since then over 19 species have been added to the genus Muscodor on the basis of morphological, volatile gas composition, phenetic, and genetic makeup from Central/ South America, Northern Territory of Australia, Thailand, China, and India (Meshram et al. 2014; Saxena et al. 2014a).

Till date, only volatile organic compounds (VOCs) produced by Muscodor have been explored and exploited for their antimicrobial, anti-insecticidal, and anti-fungal properties (Newman and Cragg 2015; Saxena et al. 2014b). Secondary metabolites of Muscodor species have not been explored extensively; there is only a single report on antimicrobial activity (Boparai et al. 2015). Hence, Muscodor species can be a novel source of new and diverse bioactive moieties which could be exploited by the pharmaceutical and the agrochemical industry.

Thus, in the present investigation, we have evaluated the in vitro xanthine oxidase inhibitory and antioxidant potential of non-volatile secondary metabolites of Indian Muscodor species.

\section{Materials and methods}

\section{Production of secondary metabolites}

Indian Muscodor species viz. Muscodor strobelii, M. darjeelingensis, M. tigerii, M. kashayum, M. ghoomensis, M. indica, and M. camphora were inoculated in potato dextrose broth for secondary metabolite production. Briefly, $5 \mathrm{~mm}$ mycelial plug of 3-4 day-old culture was inoculated into $100 \mathrm{ml}$ pre-sterilized Potato Dextrose Broth (pH 5.1) followed by incubation at $26 \pm 2{ }^{\circ} \mathrm{C}, 120 \mathrm{rpm}$ for 7 days. Subsequently, the fungal mass was separated by filtration through Whatman filter paper No. 4 followed by centrifugation at $10,000 \mathrm{rpm}$ for $10 \mathrm{~min}$. The supernatant so obtained was subjected to qualitative XOI assay.

\section{Qualitative screening of XO inhibition}

Qualitative screening of XO inhibition was carried out as per the procedure of Kapoor and Saxena (2014). The method comprised of preparation of Xanthine-Nitroblue tetrazolium (NBT) plates using $0.8 \%$ agar, $1.5 \mathrm{mg} / \mathrm{ml}$ Xanthine, and $0.11 \mathrm{mg} / \mathrm{ml} \mathrm{NBT}$. $5 \mathrm{~mm}$ wells were prepared aseptically with a sterile cork borer. Subsequently, $40 \mu \mathrm{l}$ of reaction mixture containing $30 \mu \mathrm{l}$ of each culture filtrate, $0.04 \mathrm{U}$ of xanthine oxidase (source: bovine milk), and $10 \mathrm{mmol} / \mathrm{L}$ of Tris-HCl buffer was dispensed into each well followed by overnight incubation at $37{ }^{\circ} \mathrm{C}$. The control well consisted of $30 \mu \mathrm{l}$ of un-inoculated broth and $0.04 \mathrm{U}$ of XO. Allopurinol and Febuxostat $(1 \mathrm{mM})$ were used as positive controls. Appearance of a blue-colored halo indicated the $\mathrm{XO}$ activity in control well while reduction in diameter of blue-colored halo in comparison to control-indicated XO inhibition. All the tests were carried out in triplicates. The halo diameter was recorded and data were represented as mean $\pm \mathrm{SD}$ values.

\section{Metabolite extraction from the culture filtrates}

The cell-free supernatant of each culture was extracted thrice with chloroform in the ratio of 1:2. The organic layers were pooled followed by dehydration with anhydrous sodium sulphate. The solvent was evaporated till dryness at room temperature so as to get chloroform fraction residue. The fraction so obtained was weighed and reconstituted in methanol.

\section{Quantitative estimation of xanthine oxidase inhibition}

\section{NBT assay}

The crude chloroform fractions of cultures were subjected for determination of XOI as described by Aggarwal and Banerjee (2009) with slight modifications. The crude fractions were pre-incubated with bovine milk xanthine oxidase at $37{ }^{\circ} \mathrm{C}$ for $1 \mathrm{~h}$ prior to assay. The reaction was initiated by addition of $130 \mu \mathrm{L}$ of xanthine $(10 \mathrm{mM})$ followed by $30 \mu \mathrm{l}$ of NBT. After the incubation, the amount of formazan formed was estimated by measuring the absorbance at $575 \mathrm{~nm}$ using a microplate reader (Biotek Powerwave 340, USA). Allopurinol and Febuxostat were used as positive control. Control reaction mixture consisted of substrate, enzyme, and NBT without any inhibitor. All the reactions were performed in triplicates.

\section{Uric acid estimation assay}

This assay was carried out as per the method of Chang et al. (1993), wherein the reaction mixture comprised of $10 \mu \mathrm{l}$ of crude chloroform extract and $990 \mu \mathrm{l}$ of xanthine buffer solution $(200 \mu \mathrm{M})$. The reaction was initiated by addition of $5 \mu \mathrm{l}$ of XO solution. Subsequently, the reaction mixture was mixed properly followed by incubation at $25{ }^{\circ} \mathrm{C}$ for $15 \mathrm{~min}$. The reaction was terminated by adding $1 \mathrm{~N} \mathrm{HCl}$ solution. Subsequently, the reaction was aborted by adding $1 \mathrm{~N} \mathrm{HCl}$ solution. The concentration of uric acid was measured by taking absorbance value at $290 \mathrm{~nm}$. Allopurinol and Febuxostat were used as positive controls. The percentage inhibition of xanthine oxidase was calculated by following formula:

$\%$ Inhibition $=[(A-B)-(C-D)] /(A-B) \times 100 \%$, 
where $A$ is the OD at $290 \mathrm{~nm}$ with enzyme but without sample, $B$ is the OD at $290 \mathrm{~nm}$ without sample and enzyme, $C$ is the OD at $290 \mathrm{~nm}$ with sample and enzyme, and $D$ is the OD at $290 \mathrm{~nm}$ with sample but without enzyme.

\section{Free radical scavenging activity by DPPH assay}

Antioxidant potential of the chloroform extracts of cultures were determined by the procedure of Ho et al. (2012) with minor modifications. Briefly, the reaction mixture comprised of $100 \mu \mathrm{l}$ of sample extract $(1 \mathrm{mg} / \mathrm{ml})$ mixed with $100 \mu \mathrm{L}$ DPPH solution (1, 1-diphenyl-2-picrylhydrazyl, Sigma, Final concentration $=4 \mu \mathrm{g} / \mathrm{ml}$ ). Allopurinol and Febuxostat were also evaluated for their antioxidant potential. The control comprised of $100 \mu \mathrm{l}$ of methanol and $100 \mu \mathrm{l}$ of DPPH solution. The titer plate was then incubated at $25{ }^{\circ} \mathrm{C}$ for $30 \mathrm{~min}$ in dark. The sudden decrease in absorbance was measured at $517 \mathrm{~nm}$ and the DPPH scavenging activity was calculated using the following formula:

DPPH scavenging activity $(\%)$

$=[$ Acontrol - ATest $] /$ Acontrol $\times 100$.

The test was performed in triplicates and data were represented as mean \pm SD. The antioxidant capacity was expressed as the antioxidant activity index (AAI) determined by following formula

$\mathrm{AAI}=$ final concentration of $\mathrm{DPPH}(\mu \mathrm{g} / \mathrm{ml}) / \mathrm{IC}_{50}$ of sample $(\mu \mathrm{g} / \mathrm{ml})$.

An AAI of 0.5 and below indicated a poor anti-oxidant, when AAI was between 0.5 and 1.0 it is moderate antioxidant,and when the AAI is in the range of 1.0 and 2.0 it is considered as a strong anti-oxidant, while above 2 AAI indicated a very strong anti-oxidant (Scherer and Godoy 2009).

\section{Test for purine}

The chloroform fraction of Muscodor species exhibiting the best XO inhibition was test for the presence of purine moieties using a silver precipitation test (Kapoor and Saxena 2014). Briefly the assay involved $0.5 \mathrm{ml}$ of test sample (Stock-1 $\mathrm{mg} / \mathrm{ml}$ ) in test tube and excess of ammonium hydroxide was added to it followed by addition of $0.5 \mathrm{ml}$ of $0.1 \mathrm{M}$ silver nitrate solution. Appearance of white precipitate, which is Purine-Ag+ complex, indicates the presence of purine. Allopurinol (purine analogue) was used as positive control, and Febuxostat, being non-purine in nature, served as negative control.
Table 1 Xanthine oxidase inhibition of Indian Muscodor species by qualitative and quantitative assay

\begin{tabular}{llll}
\hline Treatment & \multicolumn{2}{l}{$\%$ XO inhibition } & $\begin{array}{l}\text { \% Reduction in } \\
\text { uric acid } \\
\text { production }\end{array}$ \\
\cline { 2 - 4 } & $\begin{array}{l}\text { Xanthine } \\
\text { plate assay }\end{array}$ & NBT assay & \\
\hline Control (no inhibitor) $^{\mathrm{e}}$ & $0^{\mathrm{e}}$ & $0^{\mathrm{g}}$ & $0^{\mathrm{g}}$ \\
Febuxostat* & $59.4 \pm 1.0^{\mathrm{a}}$ & $99.2 \pm 0.0^{\mathrm{a}}$ & $99.5 \pm 0.0^{\mathrm{a}}$ \\
M. darjeelingensis & $59.4 \pm 1.0^{\mathrm{a}}$ & $91.4 \pm 0.8^{\mathrm{b}}$ & $88.1 \pm 0.0^{\mathrm{b}}$ \\
Allopurinol* $^{\mathrm{a}}$ & $58.6 \pm 1.0^{\mathrm{a}}$ & $88.0 \pm 0.5^{\mathrm{b}}$ & $86.7 \pm 0.0^{\mathrm{b}}$ \\
M. tigerii & $56.7 \pm 0.9^{\mathrm{a}}$ & $77.0 \pm 0.5^{\mathrm{c}}$ & $74.0 \pm 0.0^{\mathrm{c}}$ \\
M. kashayum & $54.9 \pm 1.0^{\mathrm{a}}$ & $76.0 \pm 1.0^{\mathrm{c}}$ & $70.3 \pm 0.0^{\mathrm{c}}$ \\
M. strobelii & $48.6 \pm 3.1^{\mathrm{b}}$ & $40.2 \pm 1.9^{\mathrm{e}}$ & $40.2 \pm 2.6^{\mathrm{d}}$ \\
M. camphora & $46.7 \pm 1.8^{\mathrm{b}}$ & $46.7 \pm 1.8^{\mathrm{d}}$ & $39.7 \pm 0.0^{\mathrm{d}}$ \\
M. ghoomensis & $31.6 \pm 3.8^{\mathrm{c}}$ & $37.6 \pm 2.7^{\mathrm{e}}$ & $24.2 \pm 0.0^{\mathrm{e}}$ \\
M. indica & $18.2 \pm 1.9^{\mathrm{d}}$ & $28.6 \pm 4.5^{\mathrm{f}}$ & $18.7 \pm 1.6^{\mathrm{f}}$
\end{tabular}

* Represent commercial inhibitors of Xanthine Oxidase (XO). All values presented are Mean \pm SD of triplicate readings

Mean values represented by same alphabets are not significantly different by Tukey's post hoc analysis at $p \leq 0.05$

\section{Results}

\section{Qualitative XO inhibition assay}

Muscodor darjeelingensis chloroform extract (CE) exhibited the highest inhibition of XO (59.4\%) all the Muscodor species tested. This was similar to the clinically approved first NP-SIXO, Febuxostat used as a standard. It was closely followed by $M$. tigerii with $56.7 \%$ inhibition which was marginally lower to allopurinol exhibiting $58.6 \% \mathrm{XO}$ inhibition in the assay. In the qualitative assay, $M$. indica CE exhibited the least XO inhibition. Febuxostat, Allopurinol, $M$. darjeelingensis $\mathrm{CE}, M$. tigerii $\mathrm{CE}$, and $M$. kashayum CE exhibited a similar XO inhibition profile in the plate assay based on Tukey's post hoc analysis (Table 1; Fig. 1).

\section{Quantitative XO inhibition}

In the quantitative NBT-based XO inhibition assay, Febuxostat exhibits $99.2 \%$ inhibition of the XO activity which was closely followed by $M$. darjeelingensis with $91.4 \%$ inhibition. Allopurinol tested as a purine-based XO inhibitor exhibited only $88 \%$ reduction in the $\mathrm{XO}$ activity during the in vitro assay. M. tigerii and M. kashayum $\mathrm{CE}$ extracts exhibited a moderate inhibition of $77 \%$ and $76 \%$, respectively. Tukey's post hoc analysis suggested that $M$. darjeelingensis $\mathrm{CE}$ and Allopurinol possessed similar potency for XO inhibition. These findings corroborated 
Fig. 1 Qualitative NBT plate assay of chloroform residues of Muscodor species

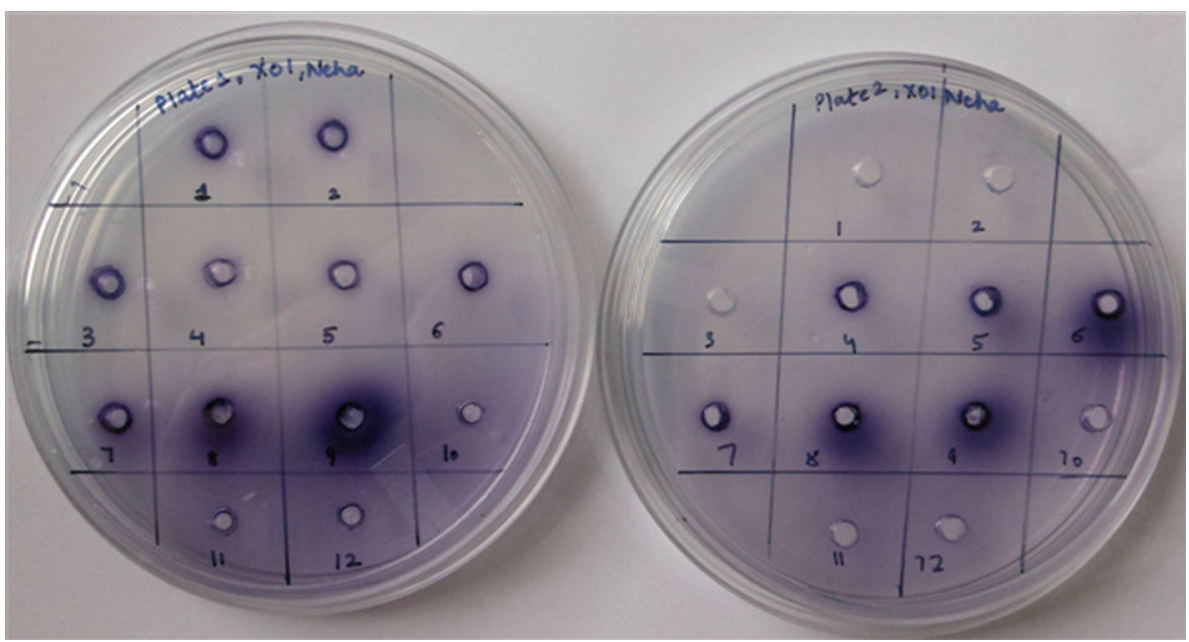

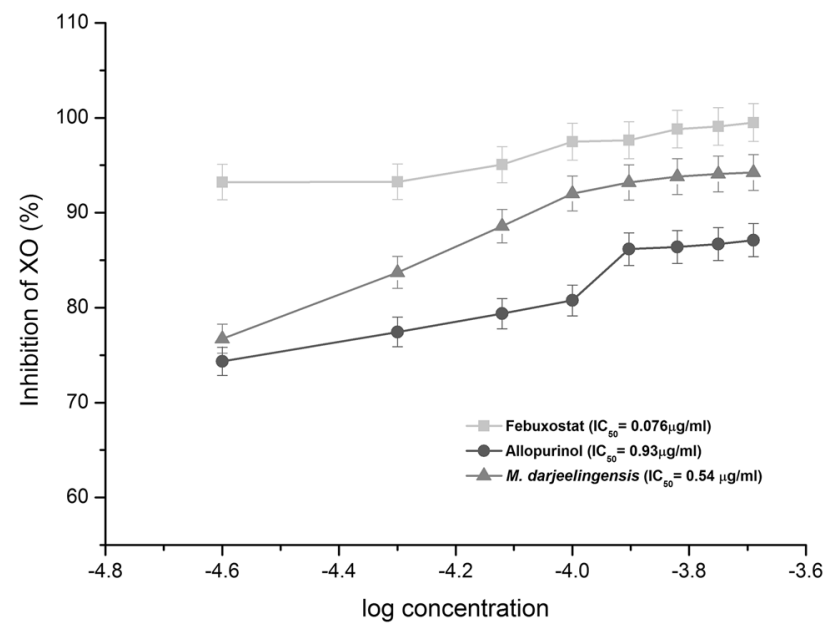

Fig. 2 Dose-response curve of chloroform residue of $M$. darjeelingensis for inhibition of xanthine oxidase. Allopurinol and Febuxostat was used as the positive control

with the reduction in uric acid production, with maximum reduction in Febuxostat, followed by $M$. darjeelingensis, Allopurinol, and M. tigerii (Table 1). Further, M. darjeelingensis $\mathrm{CE}$ exhibited an $\mathrm{IC}_{50}$ value of $0.54 \mu \mathrm{g} / \mathrm{ml}$ for $\mathrm{XO}$ in the in vitro assay (Fig. 2).

\section{Free radical scavenging assay}

Muscodor indica CE exhibited the highest free radical scavenging activity among the isolates tested and was quite similar to the Gallic acid, the standard anti-oxidant used in the DPPH assay. The $\mathrm{IC}_{50}$ value was $0.5 \mu \mathrm{g} / \mathrm{ml}$ for $M$. indica $\mathrm{CE}$ as compared to $7 \mu \mathrm{g} / \mathrm{ml}$ of Gallic acid. It also exhibited the highest AAI of 7.7 as compared to Gallic acid (Table 2; Fig. 3). M. ghoomensis CE exhibited the least antioxidant activity as well as AAI.

\section{Purine detection test}

Muscodor darjeelingensis CE did not exhibit presence of any purine moiety in the purine detection test. Febuxostat also gave a negative purine test indicating that it is a NPSIXO while Allopurinol was found to be positive for purine.

\section{Discussion and conclusion}

Hyperuricemia is a biochemical abnormality which results in gout apart from oxidative stress-related diseases. Hence, the lowering plasma uric acid levels within normal range is extremely important and can be achieved by blocking the uric acid production. Till date, only Allopurinol and Febuxostat have been clinically used for the treatment of hyperuricemia and gout; however, they have severe side effects which demand exploration of new XO inhibitors which are non- purine in nature and have lesser side effects as compared to synthetic chemicals. Endophytic fungi are relatively under tapped resources of XO inhibitors which could enter the drug discovery pipeline as anti-hyperuricemic agents. In the present study, CE of $M$. darjeelingensis was found to have a significantly lower $\mathrm{IC}_{50}$ value as compared to Fusaruside and Phenolic compounds isolated from endophytic Fusarium sp. IFB-121 and Chaetomium sp., respectively (Shu et al. 2004; Huang et al. 2007). Further phytochemicals like Isoliquiritigenin, Liquiritigenin, and Cinnamaldehyde exhibited an $\mathrm{IC}_{50}$ of 12.6, 14.29 and $8.4 \mu \mathrm{g} / \mathrm{ml}$, respectively, which was much higher than $0.54 \mu \mathrm{g} / \mathrm{ml}$ of CE of Muscodor darjeelingensis (Wang et al. 2008). Earlier, we have reported that CE of another endophytic fungus Lasiodiploda pseudotheobromae exhibited a potent $\mathrm{XO}$ inhibition with an $\mathrm{IC}_{50}$ of 
Table 2 Free radical scavenging activity by DPPH Assay

\begin{tabular}{|c|c|c|c|}
\hline Species & Scavenging activity (\%) & $\mathrm{IC}_{50}(\mu \mathrm{g} / \mathrm{ml})$ & Antioxidant activity index (AAI) \\
\hline Gallic acid (standard)* & $73.9 \pm 1.3^{\mathrm{a}}$ & 7.00 & 0.6 \\
\hline M. indica & $71.6 \pm 0.9^{\mathrm{ab}}$ & 0.52 & 7.7 \\
\hline M. strobelii & $69.8 \pm 2.6^{\mathrm{abc}}$ & 1.47 & 2.7 \\
\hline M. darjeelingensis & $68.0 \pm 3.8^{\mathrm{bc}}$ & 2.45 & 1.6 \\
\hline M. kashayum & $65.4 \pm 3.8^{\mathrm{c}}$ & 0.74 & 5.4 \\
\hline M. camphora & $48.5 \pm 3.2^{\mathrm{d}}$ & 5.64 & 0.7 \\
\hline M. tigerii & $46.5 \pm 1.7^{\mathrm{d}}$ & 47.32 & 0.08 \\
\hline M. ghoomensis & $46.4 \pm 4.9^{\mathrm{d}}$ & 133.30 & 0.03 \\
\hline
\end{tabular}

* Represent commercial inhibitors of Xanthine Oxidase (XO). All values presented are Mean \pm SD of triplicate readings Mean values represented by same alphabets are not significantly different by Tukey's post hoc analysis at $p \leq 0.05$

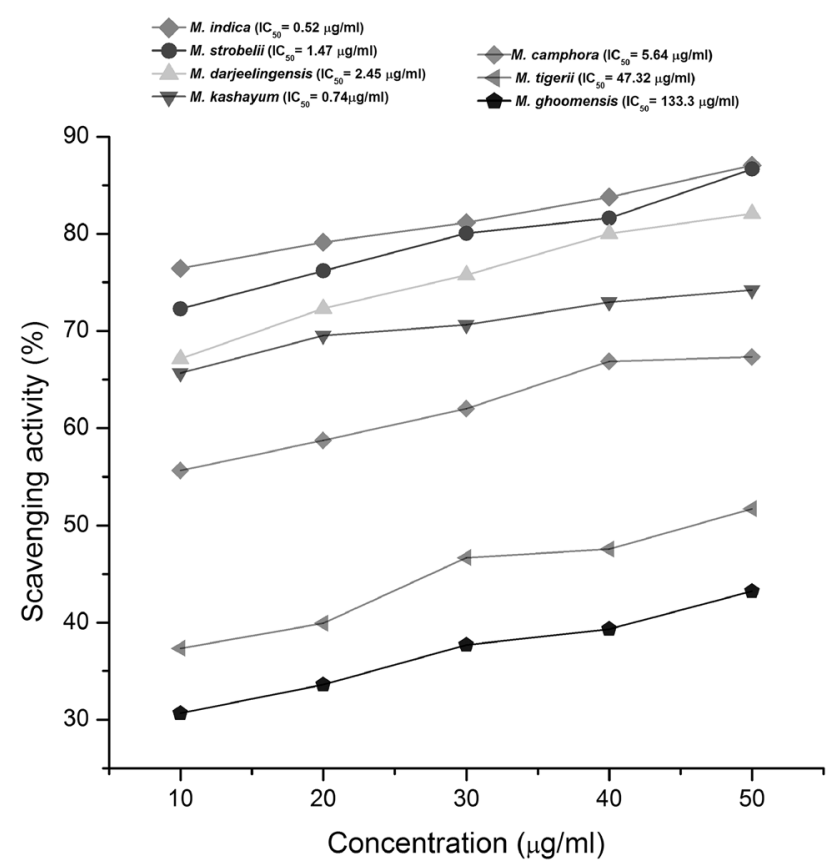

Fig. 3 Dose-response curve of chloroform residue of Indian Muscodor species for antioxidant activity by DPPH assay

$0.61 \mu \mathrm{g} / \mathrm{ml}$ (Kapoor and Saxena 2014); however, the CE of $M$. darjeelingensis exhibits a still lower $\mathrm{IC}_{50}$ for $\mathrm{XO}$ inhibition warranting potential for isolation and characterization of the bioactive moiety.

The XO inhibitory activity by endophytic fungi probably is attributed to their survival strategy to overcome the metabolically aggressive environment inside the plant. Further, they may also exhibit anti-oxidant potential to overcome the oxidative stress based defense mechanism of plants. Hence, we found that $M$. indica expressed highest anti-oxidant activity index when compared to the standard anti-oxidant Gallic acid. Muscodor tigerii and $M$. ghoomensis exhibited the least anti-oxidant activity index when compared to gallic acid. The $\mathrm{IC}_{50}$ of DPPH scavenging activity of $M$. indica was significantly low when compared to endophytic fungus Cladosporium velox TN9S isolated from T. cordifolia (Singh et al. 2016). Endophytic fungi are increasingly being explored for their antioxidant activity; however, they are seldom being reported for both anti-oxidant and Xanthine oxidase inhibitory activity which are interconnected. Hence, in the present investigation, we have tried to establish both XO inhibitory as well as anti-oxidant activity of Muscodor species isolated from India primarily from Cinnamomum sp. and Aegle marmelos. Both Cinnamomum and Aegle marmelos are medicinal plants which have been previously reported to possess anti-oxidant potential (Upadhya et al. 2004; Mathew and Abraham 2006; Jayaprakasha et al. 2006; Prasad et al. 2009; Reddy and Urooj 2013). Further, it can be hypothesized that endophytes generally mimic the medicinal properties of their host.

Thus, it can be concluded from the present study that $M$. darjeelingensis possesses a potent XOI activity while $M$. indica possesses potent anti-oxidant activity which warrants further investigation for further isolation and characterization of bioactive compounds.

Acknowledgements The Authors thank the Department of Biotechnology (National Biodiversity Development Board) for financial assistance through project no. BT/PR/10083/NDB/52/95/2007 under which this repository was created.

\section{Compliance with ethical standards}

Conflict of interest There is no conflict of interest.

Open Access This article is distributed under the terms of the Creative Commons Attribution 4.0 International License (http:// creativecommons.org/licenses/by/4.0/), which permits unrestricted use, distribution, and reproduction in any medium, provided you give appropriate credit to the original author(s) and the source, provide a link to the Creative Commons license, and indicate if changes were made. 


\section{References}

Aggarwal A, Banerjee UC (2009) Screening of xanthine oxidase producing microorganisms using nitroblue tetrazolium based colorimetric assay method. Open Biotechnol J 3:46-49

Boparai JK, Saxena S, Meshram V (2015) In vitro antimicrobial potential of Indian Muscodor species. J Basic Appl Mycol 11:22-25

Chang WS, Lee YJ, Lu FJ, Chiang HC (1993) Inhibitory effects of flavonoids on xanthine oxidase. Anticancer Res 13:2165-2170

Ho R, Violette A, Cressend D, Raharivelomanana P, Carrupt PA, Hostettmann K (2012) Antioxidant potential and radical-scavenging effects of flavonoids from the leaves of Psidium cattleianum grown in French Polynesia. Nat Prod Res 26:274-277

Huang WY, Cai YZ, Hyde KD, Corke H, Sun M (2007) Endophytic fungi from Nerium oleander L (Apocynaceae): main constituents and antioxidant activity. World $\mathrm{J}$ Microbiol Biotechnol 23:1253-1263

Jayaprakasha GK, Ohnishi-Kameyama M, Ono H, Yoshida M, Jaganmohan Rao L (2006) Phenolic constituents in the fruits of Cinnamomum zeylanicum and their antioxidant activity. J Agric Food Chem 54:1672-1679

Kapoor N, Saxena S (2014) Potential xanthine oxidase inhibitory activity of endophytic Lasiodiplodia pseudotheobromae. Appl Biochem Biotechnol 173:1360-1374

Kuo CF, Grainge MJ, Zhang W, Doherty M (2015) Global epidemiology of gout: prevalence, incidence and risk factors. Rheumatology 11:649-662

Mathew S, Abraham TE (2006) In vitro antioxidant activity and scavenging effects of Cinnamomum verum leaf extract assayed by different methodologies. Food Chem Toxicol 44:198-206

Meshram V, Kapoor N, Saxena S (2014) Muscodor strobelii, a new endophytic species from South India. Mycotaxon 128:93-104

Newman DJ, Cragg GM (2015) Endophytic and epiphytic microbes as "sources" of bioactive agents. Front Chem 3:34. doi:10.3389/ fchem. 2015.00034
Prasad KN, Yang B, Dong X, Jiang G, Zhang H, Xie H, Jiang Y (2009) Flavonoid contents and antioxidant activities from Cinnamomum species. Innov Food Sci Emerg Technol 10:627-632

Reddy VP, Urooj A (2013) Antioxidant properties and stability of Aegle marmelos leaves extracts. J Food Sci Technol 50:135-140

Saxena S, Meshram V, Kapoor N (2014a) Muscodor tigerii sp. nov.Volatile antibiotic producing endophytic fungus from the Northeastern Himalayas. Ann Microbiol 65:47-57

Saxena S, Meshram V, Kapoor N (2014b) Muscodor darjeelingensis, a new endophytic fungus of Cinnamomum camphora collected from northeastern Himalayas. Sydowia 66:55-67

Scherer R, Godoy HT (2009) Antioxidant activity index (AAI) by the 2, 2-diphenyl-1-picrylhydrazyl method. Food Chem 112:654-658

Shu RG, Wang FW, Yang YM, Liu YX, Tan RX (2004) Antibacterial and xanthine oxidase inhibitory cerebrosides from Fusarium sp. IFB-121, and endophytic fungus in Quercus variabilis. Lipids 39:667-673

Singh B, Sharma P, Kumar A, Chadha P, Kaur R, Kaur A (2016) Anti-oxidant and in vivo genoprotective effects of phenolic compounds identified from an endophytic Cladosporium velox and their relationship with host plant Tinospora cordifolia. J Ethnopharmacol. doi:10.1016/j.jep.2016.10.018

Upadhya S, Shanbhag KK, Suneetha G, Balachandra Naidu M, Upadhya S (2004) A study of hypoglycemic and antioxidant activity of Aegle marmelos in alloxan induced diabetic rats. Indian J Physiol Pharmacol 48:476-480

Wang SY, Yang CW, Liao JW, Zhen WW, Chu FH, Chang ST (2008) Essential oil from leaves of Cinnamomum osmophloeum acts as a xanthine oxidase inhibitor and reduces the serum uric acid levels in oxonate-induced mice. Phytomedicine 15:940-945

Worapong J, Strobel GA, Daisy BH, Castillo U, Baird G, Hess WM (2001) Muscodor albus anam. gen. et sp. nov., an endophyte from Cinnamomum zeylanicum. Mycotaxon 79:67-79 